\title{
Telemonitoring von Fahrrad-Heimtraining bei Patienten mit obstruktiver Schlafapnoe
}

\author{
Telemonitoring of Bike Exercise Training in Patients with \\ Obstructive Sleep Apnoea
}

Autoren

Institute
K-H. Rühle ${ }^{1,2}$, K. J. Franke ${ }^{1,2}$, U. Domanski ${ }^{1,2}$, M. Schröder ${ }^{1,2}$, G. Nilius ${ }^{1,2}$

Klinik für Pneumologie, HELIOS-Klinik Ambrock Hagen

${ }^{2}$ Universität Witten-Herdecke, Lehrstuhl Innere Medizin I eingereicht $\quad 7.3 .2013$

akzeptiert $\quad 4.4 .2013$

\section{Bibliografie}

Dol http://dx.doi.org/

10.1055/s-0033-1344151

Online-Publikation: 22.5.2013

Pneumologie 2013; 67: 321-326

(c) Georg Thieme Verlag KG

Stuttgart · New York

ISSN 0934-8387

\section{Korrespondenzadresse} Prof. Dr. med. Karl-Heinz Rühle HELIOS-Klinik Ambrock Klinik für Pneumologie Ambrockerweg 60 58091 Hagen Klinik-Ambrock.pneumo@ t-online.de

\section{Zusammenfassung \\ $\nabla$}

Hintergrund: Patienten mit obstruktiver Schlafapnoe (OSA) leiden an Tagesschläfrigkeit, kognitiven Störungen und weisen ein erhöhtes kardiovaskuläres Risiko auf. Zusätzlich zu kontinuierlich positivem Atemwegsdruck (CPAP) kann eine Veränderungen des Lebensstils mit Steigerung der körperlichen Aktivität zu einer Verbesserung des Risikos beitragen. Die tägliche Trainingsdauer sollte mindestens 20 Minuten betragen, um einen relevanten Effekt zu erzielen. Neben einem Training in Trainingsgruppen ist ein häusliches Training sinnvoll. Jedoch wird ein ausreichend intensives Training aufgrund fehlender Rückkopplung (Monitoring) häufig nicht durchgeführt. Unklar ist bis jetzt, wie viel OSA-Patienten mit einem telemonitorisch überwachten Fahrrad-Heimtraining einverstanden sind und in welchem Maße sie die für zu Hause vorgegebenen Trainingszeiten einhalten. Methodik: Von 152 konsekutiv befragten OSA-Patienten stimmten 74 (48,7\%) einem 4-wöchigen Fahrradheimtraining zu. Nach Ausschluss von 51 Patienten, 17 aus logistischen Gründen und 34 wegen gravierender Komorbiditäten, verblieben 23 Patienten (Alter 51,0+/-9,3 Jahre, BMI 33,7+/4,2, ESS-Score 10,4+/-6,7, AHI 33,8+/-24,0). Die tägliche Zeitdauer des Trainings im Bereich der ventilatorischen Schwelle (VT1) wurde mittels eines Funkmodems (Cinterion) registriert und über Internet zum Arzt oder Trainingsleiter übertragen. Ergebnisse: Die Patienten trainierten während 27,3+/-2,9 Tagen. Die Mindesttrainingsdauer von $>20$ min pro Tag erreichten 19 der 23 Patienten (83\%). Bei 4 Patienten (17\%) lag die Trainingszeit unterhalb dieser Zielmarke mit 5,9+/-2,3 min. Die durchschnittliche Trainingszeit aller Patienten lag bei 24,4+/-9,0 min.

Schlussfolgerungen: Etwa die Hälfte der Patienten mit OSA ist an einem regelmäßigen, körperlichen Fahrrad-Heimtraining mit Telemonitoring interessiert und führt dieses relativ konsequent durch.

\section{Abstract \\ $\nabla$}

Background: Patients with obstructive sleep apnoea (OSA) suffer from daytime sleepiness, cognitive disorders and are at increased risk of cardiovascular diseases. In addition to continuous positive airway pressure (CPAP), major lifestyle changes to increase physical activity contribute to risk reduction. The daily training duration should be at least 20 minutes to achieve a relevant effect. In addition to exercise training in groups, home training is useful. However, sufficiently intensive training is often not performed because of unavailable feedback (monitoring). It is not clear yet how many patients accept a bicycle home training and to what extent they meet the specified training time.

Method: Of the 152 consecutive OSA patients surveyed, 74 (48.7\%) agreed to a 4-week home exercise bike training. After exclusion of 51 patients, 17 for logistical reasons, and 34 because of severe comorbidities, 23 patients remained (age 51.0+/9.3 years, BMI $33.7+/-4.2$, ESS score $10.4+/-6.7$, AHI $33.8+/-24.0$ ). The daily duration of training at the ventilatory threshold (VT1) was recorded and transmitted by a wireless module (Cinterion) via Internet to a doctor or instructor.

Results: The patients exercised during $27.3+/-2.9$ days. 19 of the 23 patients ( $83 \%$ ) accomplished the training period of $>20$ minutes per day. In 4 patients $(17 \%)$ the training time was well below this target level with $5.9+/-2.3 \mathrm{~min}$. The average training time of all patients was $24.4+/-9.0 \mathrm{~min}$. Conclusion: About $50 \%$ of the OSA patients are interested in a regular physical exercise bike training with telemonitoring, and are performing it quite constantly. The described method of telemonitoring provides a simple and, compared to group training cost-effective way to reduce cardiovascular risk in OSA. 
Die beschriebene Telemonitoring-Methode stellt ein einfaches und im Verhältnis zu Gruppentraining kostengünstiges Verfahren zur Reduktion des kardiovaskulären Risikos bei OSA dar.

\section{Einleitung und Fragestellung}

Patienten mit obstruktiver Schlafapnoe (OSA) leiden an schlechtem Schlaf, Tagesschläfrigkeit und kognitiven Störungen und weisen ein erhöhtes kardiovaskuläres Risiko auf. Diese Störungen können oftmals nur teilweise durch eine Therapie mit kontinuierlich positivem Atemwegsdruck (CPAP) beseitigt werden.

In diesem Fall sollten zusätzlich zu CPAP weitere Therapiemodalitäten zum Einsatz kommen. Änderungen des Lebensstils mit Diät und Steigerung der körperlichen Aktivität sind einfache und effektive Methoden, die der Patient durch eigenes Zutun zur Verbesserung seines Gesundheitszustandes beisteuern kann.

Körperliches Belastungstraining über 3 Monate führt zu einer moderaten Verbesserung des Apnoe-Hypopnoe-Index (AHI) und der objektiven und subjektiven Schlafqualität [1].

Schon regelmäßige, mäßiggradige körperliche Aktivität (150 Minuten pro Woche) senkt das Risiko für Herzkreislauferkrankungen und die mit Übergewicht assoziierten Stoffwechselerkrankungen $[2,3,4]$.

Auch bei Patienten mit Herzinsuffizienz und OSA konnte durch mehrmonatiges Training die neurovaskuläre Funktion und die Schlafqualität verbessert werden [5].

Bis jetzt wurden die Patienten mit OSA in Übungsgruppen trainiert, die unter der Aufsicht eines Trainingsleiters standen [1]. Der Zeitaufwand für dieses Training ist aufgrund der z.T. langen Anfahrten sehr hoch, hinzu kommen die Kosten für den Übungsraum und den Trainingsleiter, sodass nach günstigeren Alternativen gesucht wird. Durch moderne Telekommunikationsverfahren ist es möglich, über GSM (Global System for Mobile Communication) häusliche Trainingsdaten direkt zu einer Zentrale zu senden. Die Übungszeiten z.B. mit einem Fahrrad-Heimtrainingsgerät, das beim Patienten zu Hause aufgestellt wird, können über Funk und Internet zum betreuenden Arzt übertragen werden.

Trotz der bekannten positiven Effekte hinsichtlich Schlafqualität, Stoffwechsel und Gewichtskontrolle besteht keine große Trainingsbereitschaft bei Patienten mit Übergewicht und OSA. Das Trainingsverhalten bzw. die Bereitschaft, ein Training zu beginnen, wurde bei Patienten mit OSA überprüft [6]. Von den mit der Befragung einverstandenen Patienten erklärte etwa ein Drittel, regelmäßig zu trainieren, etwa die Hälfte beabsichtigte Training in den nächsten Monaten mit unterschiedlichem Zeithorizont von Wochen bis mehreren Monaten [6]. Unklar ist bis jetzt, wie viele Patienten mit OSA mit einem telemonitorisch überwachtem Training einverstanden sind und in welchem Maße sie die für zu Hause vorgegebenen Trainingszeiten einhalten. Die Studie umfasst 2 Abschnitte:

Wir prüften 1) die Trainingsbereitschaft von konsekutiv im Schlaflabor untersuchten OSA-Patienten, bei denen erstmals ein OSA diagnostiziert wurde oder die zu einer Kontrolluntersuchung unter CPAP eingewiesen wurden, und 2) die telemonitorisch erfasste Trainingsadhärenz von Patienten in einer Untergruppe, die Trainingsbereitschaft signalisierte.

\section{Methodik \\ $\nabla$}

152 konsekutive Patienten im Alter zwischen 40-70 Jahren, 67 Patienten zur Neueinstellung auf CPAP (CE-Gruppe) und 85 Patienten, die zur CPAP-Kontrolle (CK-Gruppe) in das Schlaflabor der Klinik eingewiesen wurden, erhielten einen Fragebogen u.a. mit der Frage, ob sie grundsätzlich zu einem Fahrrad-Heimtraining bereit wären. Die Ermittlung der Trainingsbereitschaft erfolgte anhand von 3 Wahlmöglichkeiten: Einverständnis, unsicher oder Ablehnung.

Bei signalisierter Bereitschaft wurden sie dann in einem ausführlichen Beratungsgespräch über die Einzelheiten der Studie informiert. Von jeder Gruppe (CE, CK und Gesamtgruppe) standen AHI, BMI und ESS-Score zur Verfügung ( $\bullet$ Tab.1). Verglichen wurden BMI und ESS aller Patienten ohne und mit Trainingsbereitschaft.

Von den 74 trainingswilligen Patienten wurden 17 Patienten aus logistischen Gründen nicht berücksichtigt, da ihr Wohnort $>40 \mathrm{~km}$ von der Klinik entfernt war. Es verblieben 57 Patienten, von denen aufgrund einer unbehandelten Hypertonie oder gravierender Rhythmusstörungen in Ruhe und/oder Belastung weitere 34 Patienten ausgeschlossen wurden $(\bullet$ Tab. 2 ).

Somit konnten 23 Patienten (CE+CK), d.h. 40,4\% der 57 Patienten, in die Studie eingeschlossen werden $($ Tab.3)

Tab. 1 Charakteristika der 152 konsekutiven Patienten, die hinsichtlich ihrer Trainingsbereitschaft befragt wurden. CE = Patienten, die aktuell mit CPAP eingestellt worden waren. $C K=$ Patienten, die schon mehrere Monate mit CPAP behandelt wurden.

\begin{tabular}{|l|cll|} 
& $\begin{array}{l}\text { Erstdiagnose } \\
\text { (CE) } \mathbf{n = 6 7}\end{array}$ & $\begin{array}{l}\text { Kontrolle unter } \\
\text { CPAP (CK) } \mathbf{n = 8 5}\end{array}$ & $\begin{array}{l}\text { Gesamtgruppe } \\
\text { (CE + CK) } \mathbf{n = 1 5 2}\end{array}$ \\
\hline Alter (Jahre) & $53,3 \pm 13,0$ & $58,5 \pm 12,6$ & $56,2 \pm 13,0$ \\
\hline BMI & $32,3 \pm 6,2$ & $32,2 \pm 5,6$ & $32,2 \pm 5,9$ \\
\hline ESS-Score & $8,8 \pm 5,7$ & $6,2 \pm 5,6$ & $7,4 \pm 5,8$ \\
\hline AHI (Diagnose) & $26,8 \pm 22,9$ & $36,1 \pm 24,5$ & $31,7 \pm 24,1$ \\
\hline
\end{tabular}

Tab. 2 Von den 74 trainingswilligen OSA-Patienten wurden 17 aus logistischen Gründen und von den verbleibenden 54 wurden 34 Patienten aufgrund der Komorbiditäten ausgeschlossen. Es verblieben für die Trainingsstudie 23 Patienten.

\begin{tabular}{lll} 
& N & $\%$ \\
\hline $\begin{array}{l}\text { kardial: erhöhter Blutdruck, kardiale Rhythmus- } \\
\text { störungen, Z.n. kardialem Stent, manifeste } \\
\text { Herzinsuffizienz, Z.n. Herzinfarkt }\end{array}$ & 22 & 65 \\
\hline orthopädisch: Gelenkschmerzen, Rückenschmerzen & 6 & 18 \\
\hline pulmonal: Asthma bronchiale, COPD & 4 & 12 \\
\hline sonstige: Epilepsie, Thrombose & 2 & 6
\end{tabular}

Tab.3 Charakteristika der 23 Patienten, die für ein telemonitorisch überwachtes Trainingsprogramm ausgewählt wurden. CE = Patienten, die aktuell mit CPAP eingestellt worden waren. $C K=$ Patienten, die schon mehrere Monate mit CPAP behandelt wurden.

\begin{tabular}{|l|lll|}
\hline & $\begin{array}{l}\text { Erstdiagnose } \\
\text { (CE) } \mathbf{n = 1 5}\end{array}$ & $\begin{array}{l}\text { Kontrolle unter } \\
\text { CPAP (CK) } \mathbf{n = 8}\end{array}$ & $\begin{array}{l}\text { Gesamtgruppe } \\
\text { (CE + CK) } \mathbf{n = 2 3}\end{array}$ \\
\hline Alter & $50,1 \pm 9,9$ & $52,6 \pm 7,5$ & $51,0 \pm 9,3$ \\
\hline BMI & $33,5 \pm 3,7$ & $34,1 \pm 5,0$ & $33,7 \pm 4,2$ \\
\hline ESS & $8,8 \pm 6,4$ & $13,4 \pm 5,9$ & $10,4 \pm 6,7$ \\
\hline AHI (Diagnose) & $35,5 \pm 27,6$ & $30,8 \pm 17,0$ & $33,8 \pm 24,0$ \\
\hline
\end{tabular}


Tab. 4 Anzahl der OSA-Patienten mit unterschiedlicher Trainingsbereitschaft. Die Befragung erfolgte bei konsekutiv eingewiesenen Patienten des Schlaflabors. Kein relevanter Unterschied zwischen CE und CK-Patienten bzgl. Einverständnis.

\begin{tabular}{|llll|}
\hline & $\begin{array}{l}\text { Erstdiagnose } \\
\text { (CE) } \mathbf{n = 6 7}\end{array}$ & $\begin{array}{l}\text { Kontrolle unter } \\
\text { CPAP (CK) } \mathbf{n = 8 5}\end{array}$ & $\begin{array}{l}\text { Gesamtgruppe } \\
\text { (CE+CK) } \mathbf{n = 1 5 2}\end{array}$ \\
\hline $\begin{array}{l}\text { Einverständnis } \\
\text { Anzahl (\%) }\end{array}$ & $50,8 \%$ & $47,1 \%$ & $48,7 \%$ \\
\hline $\begin{array}{l}\text { unsicher } \\
\text { Anzahl (\%) }\end{array}$ & $11,9 \%$ & $3,6 \%$ & $7,2 \%$ \\
\hline $\begin{array}{l}\text { Ablehnung } \\
\text { Anzahl (\%) }\end{array}$ & $37,3 \%$ & $49,4 \%$ & $44,1 \%$ \\
\hline
\end{tabular}

Die behandlungsnaiven Patienten wurden auf eine CPAP-Therapie eingestellt, und die bereits mit CPAP behandelten Patienten setzten die Therapie fort. Alle Studienteilnehmer begannen sofort nach Entlassung das 4-wöchige Training parallel zu der nächtlichen Überdrucktherapie.

Vor dem häuslichen Training wurden alle Patienten zur Selbstsicherheit, ein Training durchzuführen, befragt. Die körperliche Aktivität vor dem Training ermittelten wir anhand des Godin Leisure Time Fragebogens (GLTEQ) [7].

Jeder Patient wurde spiroergometrisch mittels Rampenprotokoll (Steigerungsrate 10-15 Watt/min) untersucht und die Herzfrequenz an der anaeroben Schwelle (ermittelt anhand von V-Slope) bestimmt. Die ermittelte Pulsfrequenz diente dann zur Einstellung auf die ermittelte Trainingsbelastung mittels Heimtrainer (๑ Tab.4).

Häusliches Training: Das kostenlos zur Verfügung gestellte Fahrrad-Trainingsgerät war ein handelsüblicher Fahrrad-Heimtrainer, der mit einem Datenerfassungssystem und einem GSM-Modul TC 65 mit integriertem Mikro-Controller (Firma Cinterion) zur automatischen Registrierung von Trainingszeiten ausgerüstet war [8]. Die Belastung kann am Fahrradtrainingsgerät über 8 Stufen eingestellt werden, wobei mit einem Dauermagnet die Schwungscheibe abgebremst wird ( $\bullet$ Abb. 1). Mit einem berührungslosen Magnetschalter werden die Pedalbewegungen erfasst. Nach 15 Sekunden Registrierung von Impulsen durch die Pedalbewegung wird der Beginn des Trainings registriert. 15 Sekunden nach Beendigung des Trainings wird automatisch die Zeitdauer des Trainings berechnet. Die Daten wurden unter einer anonymen Patienten-Identifikation und einer SSL-Verschlüsselung per GPRS an den Server der Klinik versandt.

Die OSA-Patienten wurden gebeten, zu Hause 4 Wochen lang möglichst $30 \mathrm{~min}$ pro Tag zu trainieren, wobei ihnen bekannt war, dass die tägliche Zeitdauer des Trainings registriert und über Internet zum Arzt und Trainingsleiter übertragen wurde. Die durchschnittliche Trainingszeit pro Tag, während jeweils

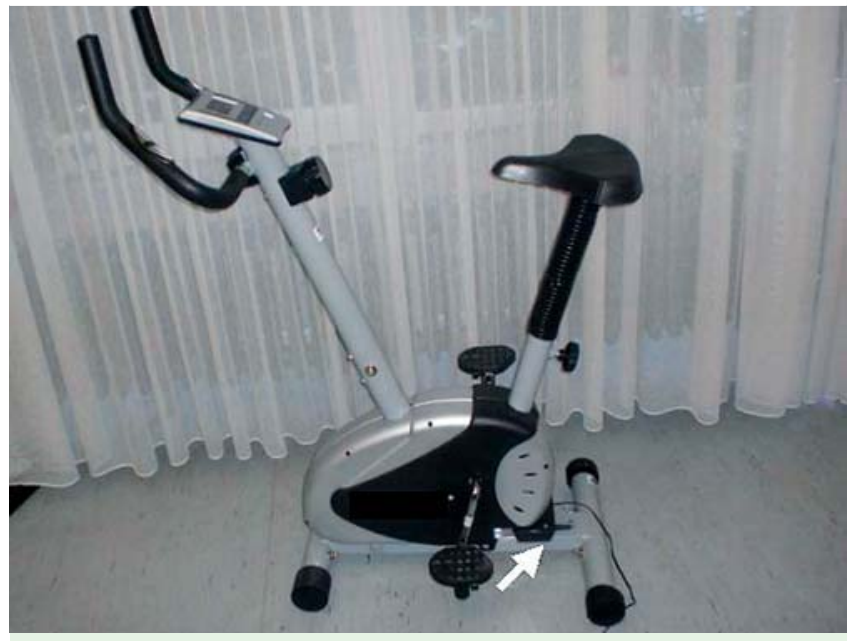

Abb.1 Fahrrad-Heimtrainingsgerät mit integriertem Mikrokontroller. Die Trainingsdauer wird automatisch über ein GSM-Modul (Global System for Mobile Communication, weißer Pfeil) 15 Sekunden nach Beendigung des Trainings verschickt.

einer Woche gemittelt, sollte $20 \mathrm{~min}$ nicht unterschreiten. Sie wurden darüber informiert, dass bei Unterschreiten der vereinbarten Zeiten ein motivierender Anruf erfolgen kann. Ermittelt wurden die täglichen Trainingszeiten (siehe $\bullet$ Abb.2). Um für zukünftige Studien einen Eindruck über die erforderliche Frequenz einer Motivation zu gewinnen, wurden die Patienten befragt, wie oft sie einen Telefonanruf zur Verbesserung der Trainingsleistung wünschen.

Die Studie wurde von der Ethik-Kommission der Universität Witten-Herdecke geprüft und genehmigt. Alle Patienten gaben ihre schriftliche Einwilligung zu der Studie.

\section{Ergebnisse}

$\nabla$

\section{Trainingsmotivation}

Von der Gesamtgruppe mit 152 Patienten stimmten 74 einem Fahrrad-Heimtraining zu, 11 waren unentschieden und 67 lehnten ein Heimtraining ab ( Tab.5). Therapie-naive Patienten und diejenigen, die bereits mit CPAP behandelt wurden, zeigten praktisch die gleiche Trainingsbereitschaft.

Beim Vergleich der Gruppen ohne und mit Trainingsbereitschaft fand sich ein signifikanter Unterschied im BMI. Die Patienten mit Bereitschaft zeigten einen signifikant höheren BMI (33,3+/-5,9 vs 31,2+/-5,5; $\mathrm{p}=0,031)$. Tendenziell zeigten die Patienten mit Bereitschaft einen höheren ESS-Score $(8,4+/-6,2$ vs 6,4+/-5,5; $\mathrm{p}=0,055)$.

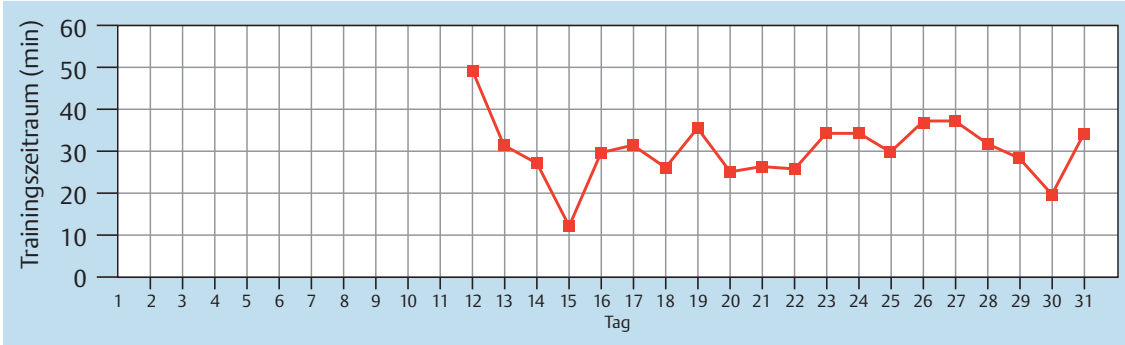

Abb.2 Diagramm der täglichen Trainingszeit im Verlauf eines Monats. Der Beginn des Trainings erfolgte am 12. des Monats. Anhand dieser Darstellung gewinnt der Trainingsleiter einen schnellen Überblick über die Trainingsaktivität. Das Beispiel zeigt eine zufriedenstellende Adhärenz während der Beobachtungszeit. 
Tab.5 Ergebnisse der Spiroergometrie-Rampenbelastung. $\mathrm{V}^{\prime} \mathrm{O}_{2}=$ Sauerstoffaufnahme $(1 / \mathrm{min}), \mathrm{HF}=$ Herzfrequenz $(1 / \mathrm{min})$, Wattpeak, $\mathrm{V} \mathrm{O}_{2}$ peak, $\mathrm{HFpeak}=$ Höchster Wert bei der symptomlimitierten Rampenbelastung $\mathrm{V}^{\prime} \mathrm{O}_{2} \mathrm{VT}$ = Sauerstoffaufnahme an der ventilatorischen Schwelle. Die Werte werden in \% des Sollwertes angegeben.

\begin{tabular}{|llllll} 
& Watt peak & V'O peak & HFpeak & HF,VT & V'O ${ }_{2}$,VT \\
\hline Mittelwert+/-SD & $161,9+/-37,9$ & $2.25+/-0.44$ & $134.6+/-17.4$ & $104.1+/-11.9$ & $1.31+/-0.25$ \\
\hline \% Soll & $81,9+/-15,1$ & $87.7+/-20.6$ & $79.2+/-8.4$ & $61.7+/-7.2$ & $49.6+/-11.9$ \\
\hline
\end{tabular}

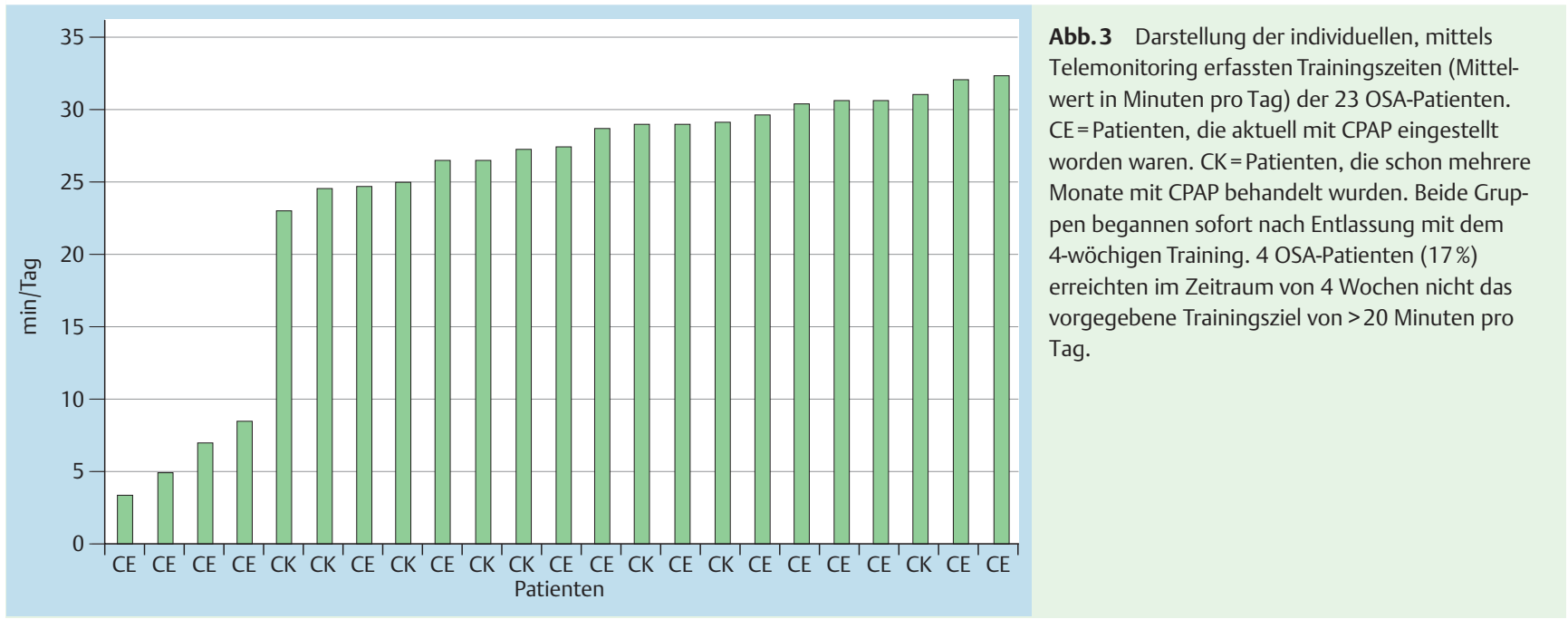

\section{Trainingsverhalten}

Vor Trainingsbeginn mit dem Godin Leisure Time Fragebogen [7] nach dem Trainingsverhalten in ihrer Freizeit in den letzten 7 Tagen befragt, berichtete die Mehrzahl der 23 Patienten (56\%) über keine oder nur leichtgradige körperliche Aktivität. Mäßiggradiges Training, wie z.B. schnelles Gehen, führten 35\% durch. Nur 9\% der Patienten trainierten bereits mit hoher Herzfrequenz.

Fast alle eingeschlossenen Patienten zeigten eine positive Einstellung zum geplanten Training. Bei etwa der Hälfte aller Patienten bestand zu Trainingsbeginn eine gewisse Skepsis hinsichtlich des Trainings allein zu Hause. 13 von 23 gaben an, dass das Trainieren allein zu Hause keine Freude macht.

Die während der Rampenbelastung erreichte Peak $\mathrm{V}^{\prime} \mathrm{O}_{2}$ lag im Mittel bei 87,7+/-20,6\% des Referenzwertes. Die an der ventilatorischen Schwelle (anaeroben Schwelle) erreichte Herzfrequenz lag bei 104,1+/-11,9 (siehe auch $\bullet$ Tab.4).

Die Patienten trainierten telemonitorisch überwacht während $27,3+/-2,9$ Tagen. Die Mindesttrainingsdauer von $>20$ min pro Tag erreichten 19 der 23 Patienten (83\%). Bei 4 Patienten (17\%) lag die Trainingszeit unterhalb dieser Zielmarke mit 5,9+/-2,3 min $(\bullet$ Abb.3). Die durchschnittliche Trainingszeit aller Patienten lag bei $24,4+/-9,0 \mathrm{~min}$.

Auf die Frage, wie häufig ein Telefongespräch/ein Anruf durch den Trainingsleiter erfolgen sollte, wünschten 10 Patienten einen wöchentlichen Anruf, 5 alle 2 Wochen, 5 alle 4 Wochen und 2 alle 12 Wochen. Nur ein Patient hielt Anrufe zu Hause für nicht erforderlich.

\section{Diskussion}

In dieser Studie berichten wir unseres Wissens nach zum ersten Mal über Fahrrad-Heimtraining mit Telemonitoring der Trainingszeiten bei einer OSA-Patientengruppe.
Belastungs-Training spielt eine wesentliche Rolle bei der Erhöhung der Leistungsfähigkeit und der Verminderung des kardiovaskulären Risikos von Patienten mit OSA. Im Folgenden sollen die positiven Effekte körperlicher Aktivität bei OSA kurz dargestellt werden: Körperliches Training führte in verschiedenen Studien, allerdings mit geringen Patientenzahlen, zur Reduktion des AHI bei praktisch konstantem Körpergewicht. Durch das körperliche Training wurde der durchschnittliche AHI von 33/h auf 24/h, d.h. um 27\% vermindert [9]. In einer weiteren Studie an 9 OSA-Patienten fiel der AHI nach zwei Stunden wöchentlichen Trainings von 22 auf $12 / \mathrm{h}$ [10].

Die Verbesserung der schlafbezogenen Atmungsstörung (SBAS) kann eventuell durch eine Gewichts-Umverteilung bzw. des Fettgewebes im Rahmen der vermehrten körperlichen Aktivität erklärt werden [11]. Kline et al. [1] zeigten nach einem 12-wöchigen Training bei $60 \%$ der Herzfrequenzreserve eine Reduktion des AHI ohne relevante Veränderung des BMI. Auch die objektive und subjektive Schlafqualität verbesserte sich. Da etwa $30 \%$ aller Patienten mit OSA trotz Therapie mit CPAP über weiterhin bestehende Tagesschläfrigkeit klagen, sollte an eine zusätzliche Trainingstherapie gedacht werden. Wöchentliches Training mit $>3$ Stunden körperlicher Aktivität reduzierte die odds ratio, an einer SBAS mit einem AHI > 15 zu erkranken, auf 0,68 [12].

Die Lebenserwartung von Patienten mit unbehandeltem OSA ist mit einem erheblichen kardiovaskulären Risiko behaftet $[13,14$, 15]. Viele dieser Patienten leiden an einem metabolischen Syndrom infolge des erheblichen Übergewichts. Das metabolische Syndrom (MetS) ist mit der Entwicklung von Diabetes mellitus und kardiovaskulären Erkrankungen assoziiert, wobei ein 2,9fach höheres Risiko, an einer Herz-Kreislauferkrankung zu versterben, resultiert [16].

Die Kombination von OSA und MetS erhöht das Risiko durch Anstieg des Blutdrucks, Zunahme des sympathischen Drive und Verminderung des Baroreflexes [17]. 
Die Häufigkeit des metabolischen Syndroms lag in einer Untersuchung von Patienten mit ausgeprägter Obesitas ohne OSA bei $36 \%$ und stieg bei zusätzlichem OSA auf das Doppelte an [18]. Die Therapie mit CPAP verbessert den Lipidstoffwechsel mit Erniedrigung der Spiegel für Gesamtcholesterin, Triglyceride, LDL und Erhöhung des HDL-Cholesterins und Insulinresistenz. Durch eine CPAP-Therapie wird das kardiovaskuläre Risiko durch die oben genannten Effekte von CPAP zwar reduziert, aber lediglich bei $20 \%$ der Patienten bildete sich das MetS zurück [19], sodass zusätzliche Maßnahmen zur Verbesserung des Zucker- und Fettstoffwechsels, wie z.B. körperliche Bewegung/Training, erforderlich sind.

Die Hauptbefunde dieser Studie sind (1) etwa die Hälfte der Patienten, denen ein häusliches Training mit einem Fahrrad-Heimtrainer angeboten wurde, war zu einem telemonitorisch überwachten Training bereit; (2) bei etwa $40 \%$ dieser Patienten war bei relativ strengen Ausschlusskriterien wegen Begleiterkrankungen ein Heimtraining möglich; (3) von 81\% der ausgewählten OSA-Patienten wurde die vorgeschriebene Trainingszeit von mehr als 20 Minuten pro Tag während eines 4-wöchigen Heimtrainings erreicht.

$\mathrm{Zu}$ (1): Die Motivation zum körperlichen Training eines unselektierten OSA-Patientenguts eines Schlaflabors mit zugewiesenen Patienten war mit etwa $50 \%$ überraschend hoch. Dies dürfte damit zusammenhängen, dass ein konkretes Trainingsangebot gemacht und das Trainingsgerät kostenlos zur Verfügung gestellt wurde. Im Vergleich zu Smith [6] (37\% aktives Training) waren die meisten unserer Patienten vorher nicht trainingsaktiv (9\%). Höherer BMI und höherer ESS-Score in der motivierten Gruppe weisen in unserer Untersuchung darauf hin, dass ein größerer Leidensdruck (Übergewicht und Tagesschläfrigkeit) die Trainingsbereitschaft fördert. Im Vergleich zu OSA-Patienten ist die Trainingsbereitschaft von COPD-Patienten etwas geringer [8].

$\mathrm{Zu}$ (2): Da das Training ohne Überwachung der Vitalparameter zu Hause erfolgte, waren unsere Auslesekriterien besonders streng. Deshalb mussten fast $60 \%$ von dem Training ausgeschlossen werden. Denkbar wäre, dass auch Patienten mit höheren Risiken bei zusätzlichem Monitoring von Puls und $\mathrm{O}_{2}$-Sättigung und engerer ärztlicher, ambulanter Überwachung zu Hause trainieren können. In der Regel überwiegt der Nutzen eines körperlichen Trainings die gleichzeitig bestehenden Risiken.

Zu (3): In unserer Studie erreichten über 80\% der Patienten die für einen ausreichenden metabolischen Effekt erforderlichen Trainingszeiten. Allerdings bezieht sich dieser Prozentsatz auf lediglich 4 Wochen Trainingszeit, bei Langzeitanwendung dürfte die Adhärenz eventuell absinken.

Diese hängt entscheidend von der internen und externen Motivation und den Belohnungsmechanismen ab. Wird das Training abgebrochen, geht der Effekt innerhalb weniger Wochen auf das Ausgangsniveau zurück und die Stoffwechselstörung bzw. das Risiko verschlechtern sich. Es ist deshalb entscheidend, dass ein nachhaltiges Training erfolgt. Die meisten Trainingsprogramme scheitern, weil regelmäßiges Training zu Hause nicht gewährleistet ist. Gemeinsames Training in ambulanten Sportgruppen ist für den Einzelnen zeitaufwändig und findet selten mehr als zweimal pro Woche statt. Durch Telemonitoring kann die Mitarbeit des Patienten auch zu Hause registriert und die Abnahme der Adhärenz durch telefonische Kommunikation verbessert werden. Die online aufgezeichneten Trainingszeiten stehen täglich zur Verfügung. Bei adhärenten Patienten ist es nicht nötig, diese telefonisch zu motivieren. Bei nicht adhärenten Patienten kann zeit- nah die Ursache des verminderten oder abgebrochenen Trainings telefonisch eruiert bzw. zu längerer Leistung motiviert werden. Die meisten unserer Patienten waren mit denjenigen der Studie von Kline [1] bezüglich Lebensstil mit wenig Bewegung und Übergewicht vergleichbar. Unterschiede ergeben sich hinsichtlich Motivation und Rekrutierung: Während in unserer Studie nach 4 Wochen $20 \%$ der Patienten abbrachen, beendeten bei Kline [1] nur 10\% (3 von 27) bei einem 12 Wochen dauernden Protokoll das Training. Sie waren neben der Motivation durch Gruppentraining durch eine Prämie von $300 \$$ nach Abschluss der Studie besonders motiviert.

Eine der Limitationen unserer Studie besteht darin, dass die Patienten lediglich während eines Zeitraums von 4 Wochen trainierten. Ein Effekt bzgl. Hämodynamik und Schlafqualität ist nach einer solch kurzen Periode nicht zu erwarten. Der Schwerpunkt unserer Untersuchung lag vielmehr auf der Trainingsbereitschaft von OSA-Patienten und der initialen Adhärenz eines telemonitorisch überwachten häuslichen Trainings.

Zusammenfassend hat diese Studie gezeigt, dass etwa die Hälfte der Patienten mit OSA an einem regelmäßigen, körperlichen Training interessiert ist. Nach Ausschluss von Kontraindikationen verbleibt etwa die Hälfte, d.h. also etwa ein Viertel aller angesprochenen Patienten mit OSA. Der größte Teil dieser Patienten erreichte die vorgeschriebene Trainingsmindestdauer von $20 \mathrm{Mi}$ nuten.

\section{Schlussfolgerungen}

Der Vorteil des hier vorgestellten Verfahrens beruht auf einer Technik, die keine Mitarbeit der Patienten bei der Messung der Trainingszeiten erfordert. Da die Trainingszeiten sofort zur Verfügung stehen, kann bei Bedarf eingeschritten werden, wenn die Trainingsadhärenz abnimmt. Telemonitoring von Heimtraining stellt eine einfache und im Verhältnis zum Gruppentraining kostengünstige Methode dar. Damit dürfte es in Zukunft möglich sein, mehr Patienten mit OSA in Trainingsprogramme zur Reduktion von Morbidität und Mortalität einzubinden.

\section{Interessenkonflikt}

\section{$\nabla$}

K.-H. Rühle und G. Nilius erhielten finanzielle Unterstützung von Fisher \& Paykel Healthcare, Heinen und Löwenstein, ResMed, TNI, Vitaphon und Weinmann. Der Artikel wurde finanziell nicht unterstützt. Karl-Josef Franke, Ulrike Domanski and Maik Schröder geben keinen Interessenkonflikt an.

\section{Literatur}

1 Kline CE, Crow IP, Ewing GB et al. The effect of exercise training on obstructive sleep apnea and sleep quality: a randomized controlled trial. Sleep 2011; 34: 1631 - 1640

2 Wen CP, Wai JP, Tsai MK et al. Minimum amount of physical activity for reduced mortality and extended life expectancy: a prospective cohort study. Lancet 2011; 378: 1244-1253

3 Marwick TH, Hordern MD, Miller T et al. Exercise training for type 2 diabetes mellitus: impact on cardiovascular risk: a scientific statement from the American Heart Association. Circulation 2009; 119: 3244 3262

4 Tjønna AE, Lee SJ, Rognmo Ø et al. Aerobic interval training versus continuous moderate exercise as a treatment for the metabolic syndrome: a pilot study. Circulation 2008; 118: $346-354$

5 Ueno LM, Drager LF, Rodrigues AC et al. Effects of exercise training in patients with chronic heart failure and sleep apnea. Sleep 2009; 32: $637-647$ 
6 Smith SS, Doyle G, Pascoe T et al. Intention to exercise in patients with obstructive sleep apnea. J Clin Sleep Med 2007; 3: 689-694

7 Godin G, Shephard RJ. A simple method to assess exercise behavior in the community. Can J Appl Sport Sci 1985; 10: 141-146

8 Rühle KH, Bloch M, Franke KJ et al. Telemonitoring of arm ergometric training in COPD patients. A pilot study. Pneumologie 2009; 63: $314-$ 318

9 Giebelhaus V, Strohl KP, Lormes $W$ et al. Physical exercise as an adjunct therapy in sleep apnea - An open trial. Sleep Breath 2000; 4: 173-176

10 Norman JF, Von Essen SG, Fuchs RH et al. Exercise training effect on obstructive sleep apnea syndrome. Sleep Res Online 2000; 3: 121-129

11 Peppard PE, Young T. Exercise and sleep-disordered breathing: an association independent of body habitus. Sleep 2004; 27: 480-4

12 Quan SF, O'Connor GT, Quan JS et al. Association of physical activity with sleep-disordered breathing. Sleep Breath 2007; 11: 149-157

13 Young T, Finn L, Peppard PE et al. Sleep disordered breathing and mortality: eighteen-year follow-up of the Wisconsin sleep cohort. Sleep 2008; 31: $1071-1078$
14 Marshall NS, Wong KK, Liu PY et al. Sleep apnea as an independent risk factor for all-cause mortality: the Busselton Health Study. Sleep 2008; 31: $1079-1085$

15 Gottlieb DJ, Yenokyan G, Newman AB et al. Prospective study of obstructive sleep apnea and incident coronary heart disease and heart failure: the sleep heart health study. Circulation 2010; 122: 352-360

16 Lakka HM, Laaksonen DE, Lakka TA et al. The metabolic syndrome and total and cardiovascular disease mortality in middle-aged men. JAMA 2002; 288: 2709 - 2716

17 Trombetta IC, Somers VK, Maki-Nunes C et al. Consequences of comorbid sleep apnea in the metabolic syndrome - implications for cardiovascular risk. Sleep 2010; 33: 1193-1199

18 Gasa M, Salord N, Fortuna AM et al. Obstructive sleep apnoea and metabolic impairment in severe obesity. Eur Respir J 2011; 38: 1089 1097

19 Sharma SK, Agrawal S, Damodaran D et al. CPAP for the metabolic syndrome in patients with obstructive sleep apnea. N Engl J Med 2011; 365: $2277-2286$ 